\title{
Liquid core integrated ring resonator
}

\author{
Genni Testa ${ }^{\mathrm{a}}$, Yujian Huang ${ }^{\mathrm{c}}$,Luigi Zeni ${ }^{\mathrm{b}}$, Pasqualina M. Sarro ${ }^{\mathrm{c}}$, Romeo Bernini ${ }^{\mathrm{a}^{*}}$ \\ ${ }^{a}$ IREA-CNR,Via Diocleziano 328, 80124 Napoli, Italy \\ ${ }^{b}$ DII, Seconda Università di Napoli, Via Roma 29, 81031 Aversa. \\ 'ECTM-DIMES, TUDelft, Feldmannweg 17, NL-2600 GB Delft, The Netherlands.
}

\begin{abstract}
We report the design and the fabrication of an integrated optofluidic ring resonator based on liquid core antiresonant reflecting optical waveguide (ARROW) operating around $\lambda=635 \mathrm{~nm}$ with a water filled core $(\mathrm{nc}=1.33)$. This waveguide permits a strong coupling between light and analyte as the same microchannel can be used for delivering sample and for confining light. A rectangular-shaped ring resonator has been realized by using four 90-bent waveguides and an multimode interference coupler is used for coupling light from the input bus waveguide to the resonator. The resulting device is very compact, with a total length of $2.5 \mathrm{~mm}$ and a required liquid volume of about $0.11 \mathrm{nl}$. The device has been simulated with a two dimensional finite-difference time-domain method for different refractive index core $\mathrm{n}_{\mathrm{c}}=1.32$ (methanol) and $\mathrm{n}_{\mathrm{c}}=1.43$ (dimethylformamide). Moreover, we carried out optical measurements by measuring the transmitted spectrum from the output bus waveguide in both cases. The experimental results confirm that ring with a good quality factor $(\mathrm{Q} \sim 800)$ can be achieved in good agreement with the numerical results.
\end{abstract}

Keywords: Ring resonator, Optofluidics, Liquid core waveguides, Optical sensors

\section{INTRODUCTION}

In the field of guided-wave optical biosensor, ring resonators are of great interest due to their high sensitivity. For this reason several devices geometries as microspheres [1], microrings [2] and microdisks [3] have been widely investigated for application in sensing area. The high sensitivity of these devices relies on the interaction length between the light and sample that is not limited by the physical length but is related to the number of revolutions of light in the resonator, characterized by the quality factor Q. In fact, unlike the other integrated biosensors, in a ring resonator the circulation of light in the resonator enables light to interact multiple times with the sample, in this way approaching to single-molecule detection limit.

Basically, when applied in the fields of optical biochemical sensing, all the resonator-based sensors proposed are based on two major sensing mechanisms: refractive index sensing [1,3], and absorption sensing [4]. High sensitivities for homogeneous or surface sensing are possible by optimizing waveguide parameters; in particular the sensitivity of an optical waveguide sensor relies on the amount of light in the medium to be sensed. For homogeneous sensing, the main design goal is to find suitable design able to maximize the distribution of the optical field in the sample to be measured. In order to achieve high performance these structures requires an optimized integration of the optical and microfluidic parts. In many cases the design of optical part has been privileged respect to microfluidic one, sacrificing the feasibility and the multiplexing capability of the device. Recently, a great effort has been devoted to find new integration strategies like liquid core ring resonator [5] or to realize effective fluidics in high sensitivity integrated ring resonator [6]. However, until now a fully integrated ring resonator has not yet proposed.

In this work we report an optofluidic ring based on liquid core antiresonant reflecting optical waveguide (ARROW). The advantage of using thse waveguides relies on the possibility to use the same microchannel for guiding light and for delivering the sample to be sensed. In this way the interaction efficiency between the propagating optical mode and the sample is optimized. Moreover, using these waveguides the microfluidic and the optical parts are integrated to form a very compact device. However, ARROW waveguides are leaky waveguides, and for this reason an accurate design is necessary in order to minimize the optical losses and achieve good performances of the ring.

*bernini.r@irea.cnr.it, phone+39081570799, fax:+390815705734,www.irea.cnr.it. 
The optical response of the device has been simulated with two dimensional finite-difference time-domain method and compared with the experimental ones. We have simulated and characterized two configurations for the optical ring, obtained by filling the core with two different fluid materials having refractive index $\mathrm{n}_{\mathrm{c}}=1.32$ (methanol) and $\mathrm{n}_{\mathrm{c}}=1.43$ (dimethylformamide). From the measured and simulated transmitted spectrum we have derived the free spectral range and the quality factor of the ring for both configurations. The numerical and the experimental results were found to be in very good agreements. Also the sensing capabilities of the ring have been derived from measurements.

\section{DEVICE DESIGN AND FABRICATION}

The proposed ring resonator is based on liquid core antiresonant reflecting optical waveguides (ARROWs) [7,8]. By using liquid waveguides we are able to use the same channel for guiding light and, at the same time, for delivering the sample under analysis; this results in a strong liquid-light interaction with great potential in sensing application.

The layout of the proposed device is shown in Fig. 1. Four $90^{\circ}$-bent ARROW waveguides are used to form a rectangular ring resonator and a multimode interference (MMI) liquid core ARROW coupler with a 50:50 splitting ratio acts as coupling acts as coupling element between the ring and the bus waveguide. The choice of $90^{\circ}$-bent ARROW waveguides permits to reduce the propagation losses due to the bend [9] and hence to improve the ring quality factor. The MMI coupler has a width of $\mathrm{W}_{\mathrm{MMI}}=4 \mu \mathrm{m}$ and it has been designed to operate as a balanced power splitter at the working wavelength of $\lambda=635 \mathrm{~nm}$ for a water-filled liquid core $\left(\mathrm{n}_{\mathrm{c}}=1.33\right)$. This results in a length of $\mathrm{L}_{\mathrm{MMI}}=2 \mathrm{n}_{\mathrm{c}} \mathrm{W}^{2}{ }_{\text {MMI }} / \lambda=67.17 \mu \mathrm{m}$. Moreover, for increasing the coupling efficiency with single mode optical fibers, we used linear tapered waveguides to connect the input and output ports of the MMI coupler with input and output straight access waveguides of $5 \mu \mathrm{m}$ width. The round-trip length of the ring resonator is $\mathrm{L}_{\mathrm{RT}}=174.4 \mu \mathrm{m}$ while the total length of the device, including the input and output access waveguides, is $\mathrm{L}=2.5 \mathrm{~mm}$. The total liquid volume required is about $0.11 \mathrm{nl}$. A scanning electron microscope (SEM) image of the ring resonator is shown in Fig. 2.

The ARROW waveguide has been designed to minimize the propagation loss for a water-filled core $\left(\mathrm{n}_{\mathrm{c}}=1.33\right)$ at a wavelength of $\lambda=635 \mathrm{~nm}$. Light propagation in these waveguides occurs within the inner liquid-filled core, thanks to a surrounding multilayer stack designed to operate as a high reflectivity Fabry-Perot (F-P) mirror [7, 8]. The ARROW structure is composed by two half of silicon wafer substrates, the first fabrication step consists in a photolithographic process and reactive dry etching to define the core channel on the bottom wafer. The transverse section of the integrated liquid core ARROW waveguide is shown in Fig. 3. In agreement with the well-known optical properties of the ARROWs [8], the single mode operation of the device has been assured by choosing the appropriate geometrical parameters for the waveguide's core: a transverse core thickness of $d_{c}=10 \mu \mathrm{m}$ and a with core widths $\mathrm{d}=2 \mu \mathrm{m}$. This configuration supports a TE polarized optical mode with respect to the lateral confinement. The F-P mirrors are composed by two alternating layers of titanium dioxide $\left(\mathrm{TiO}_{2} . \mathrm{n}_{1}=2.49\right)$ and silicon dioxide $\left(\mathrm{SiO}_{2}, \mathrm{n}_{2}=1.46\right)$. The deposition of a conformal silicon dioxide (TEOS) $\mathrm{SiO}_{2}$-layer is carried out by low pressure chemical vapour deposition (LPCVD) technique on both wafers, at a temperature of $850^{\circ} \mathrm{C}$. The fabrication process is then completed by depositing, on both wafers, the titanium dioxide cladding layer by using the atomic layer deposition (ALD) process. The thicknesses of $\mathrm{d}_{1}=75.4 \mathrm{~nm}\left(\mathrm{TiO}_{2}\right)$ and $\mathrm{d}_{2}=262 \mathrm{~nm}(\mathrm{SiO} 2)$ of the cladding layers are properly chosen to operate to the first order of antiresonance.

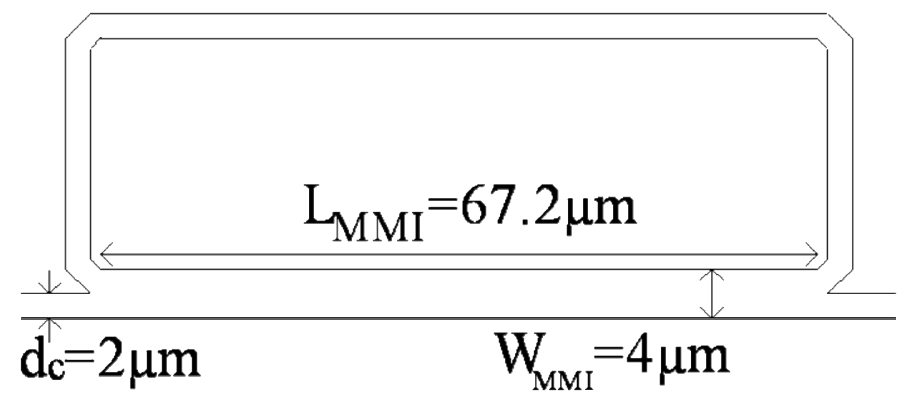

Fig. 1 Schematic drawing of the ring resonator. 


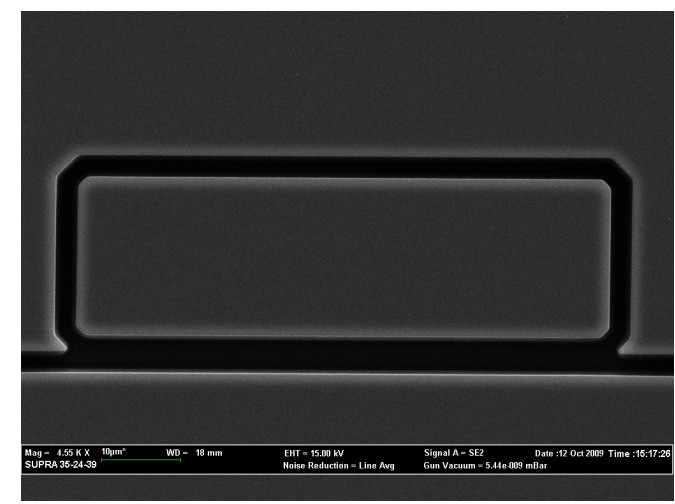

Fig. 2 SEM picture of the fabricated device.

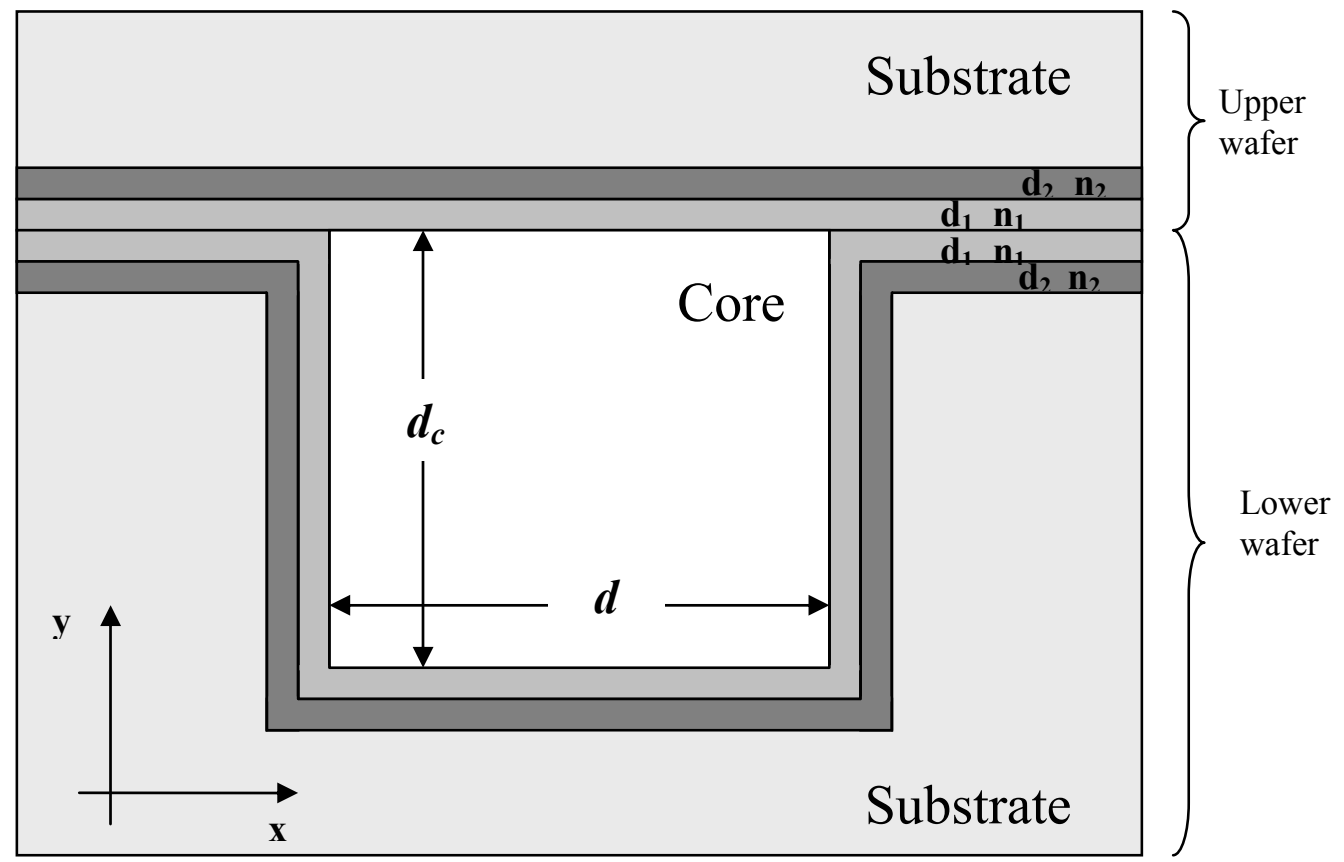

Fig.3 Liquid core ARROW waveguide transverse section.

\section{DEVICE SIMULATION}

The performance of the device has been simulated using a two dimensional finite-difference time-domain method (FDTD) (OmniSim, OPhoton Design). The core refractive index has been set to $\mathrm{n}_{\mathrm{c}}=1.32$ (methanol). We assumed as input field the fundamental TE-mode of a $2 \mu \mathrm{m}$ wide ARROW waveguide at $\lambda=640 \mathrm{~nm}$. The mode profile was calculated with a 2D mode solver (FIMMWAVE, (C) Photon Design). Moreover, in order to analyze the spectral response of the resonator, we have modeled the input field as a light pulse centered at $\lambda=640 \mathrm{~nm}$ with a full width at half maximum (FWHM) linewidth of $\Delta \lambda_{\mathrm{FWHM}}=5 \mathrm{~nm}$. The performance of the MMI at the operation wavelength of $\lambda=640 \mathrm{~nm}$ and $\mathrm{n}_{\mathrm{c}}=1.32$ has been previously evaluated in order to understand the deviation from the expected performance. We have evaluated that these parameters leads to a very weak unbalancing (49.6:50.4) between the two output ports of the MMI.

The spectral information has been derived from simulation results by performing a discrete Fourier transform of the time domain data. Due to the constructive interference in the ring, resonances can be observed in the normalized transmitted spectrum at the output of the bus waveguide. From the simulated spectrum (Fig.4) we have calculated a free spectral range (FSR) of FSRs $\sim 1.65 \mathrm{~nm}$ at around $\lambda=640 \mathrm{~nm}$ and an extinction ratio (ER) ERs $\sim 5.74 \mathrm{~dB}$. The quality factor of the ring is $Q s \sim 1150$. 


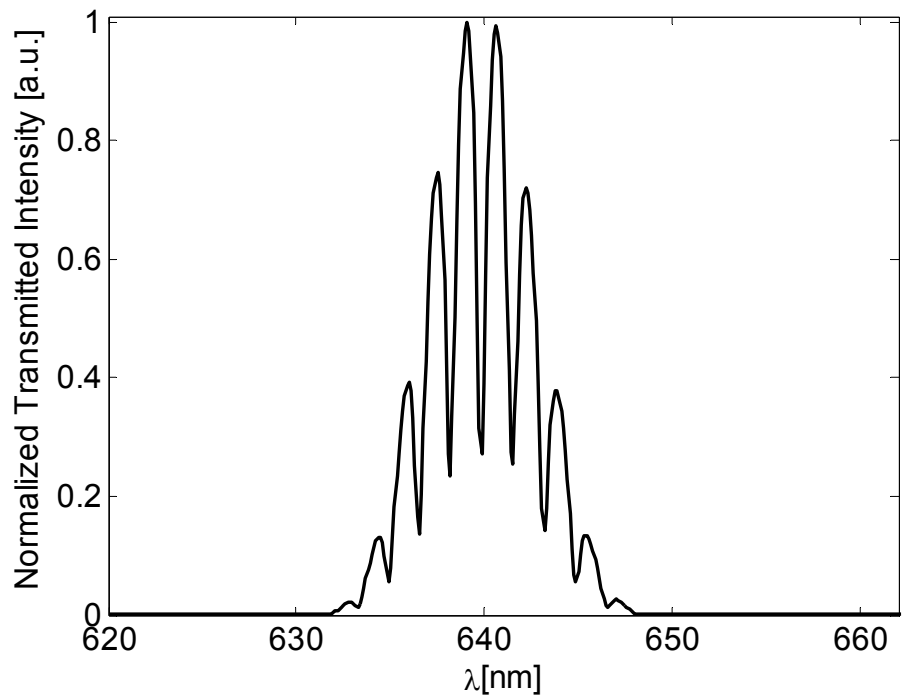

Fig. 4 Simulated transmitted intensity for methanol filled ring resonator $\left(\mathrm{n}_{\mathrm{c}}=1.32\right)$.

The steady-state response of the resonator at a resonance wavelength has been simulated with FDTD method. In Fig.5(a) the spatial profiles of the light intensity in the ring resonator at the resonance wavelength for methanol filled core $\left(\mathrm{n}_{\mathrm{c}}=1.32\right)$ is reported. For comparison we have also simulated the propagation of light in the device at an off-resonance wavelength (Fig.5(b)).

In order to demonstrate the possibility of further improvement of the ring performance we have also simulated the spectral response of the device with $\mathrm{n}_{\mathrm{c}}=1.43$ (dimethylformamide (DMF) refractive index). This is due to the fact that in an ARROW waveguides the propagation losses can be lowered by filling the core with a higher refractive index fluid. In this way it is possible to improve the overall optical properties of the ring resonator. The simulated spectrum obtained by modeling the device with $\mathrm{n}_{\mathrm{c}}=1.43$ is shown in Fig.6. From the spectrum we have estimated ERs $\sim 7.65 \mathrm{~dB}$ and FSRs $\sim 1.53$ $\mathrm{nm}$. In this case we have calculated a quality factor of Qs 1380 and hence a ring with better performance.

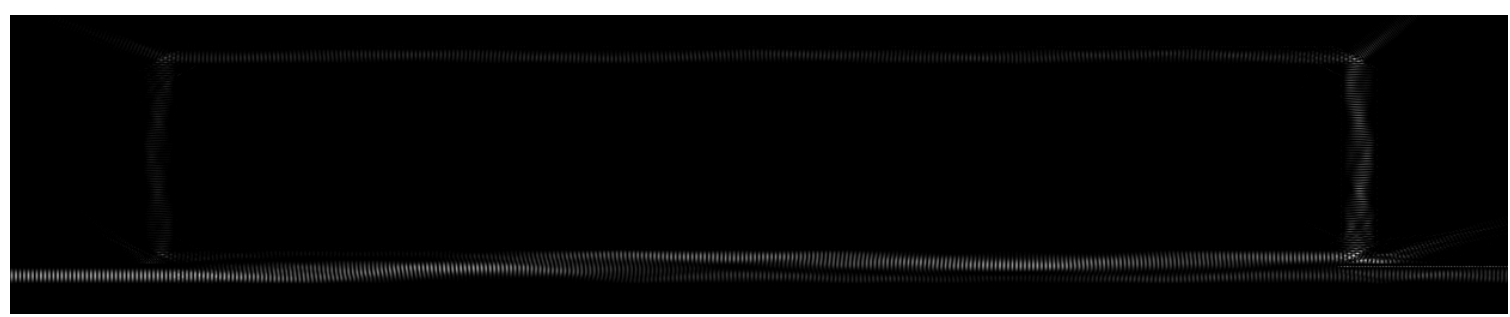

(a)

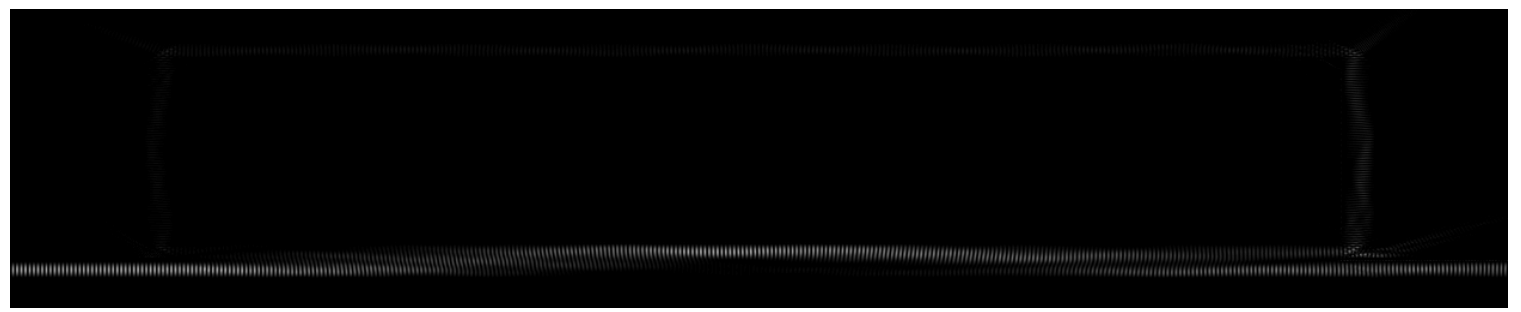

(b)

Fig. 5 (a) Spatial profiles of the light intensity in the ring resonator at the resonance wavelength for methanol filled core ( $\left.n_{c}=1.32\right)$; (b) Spatial profiles of the light intensity in the ring resonator off-resonance condition for methanol filled core $\left(\mathrm{n}_{\mathrm{c}}=1.32\right)$. 


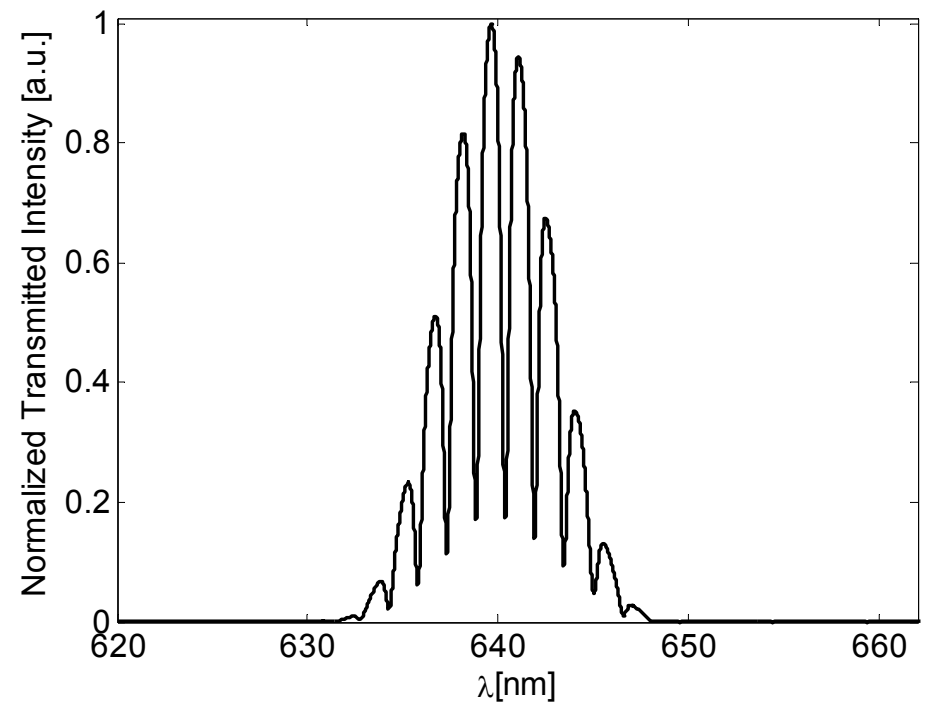

Fig. 6 Simulated transmitted intensity for DMF filled ring resonator $\left(\mathrm{n}_{\mathrm{c}}=1.43\right)$

\section{EXPERIMENTAL RESULTS}

The optical characterization has been carried out by coupling the light from a laser diode at $\lambda=640 \mathrm{~nm}$ (FWHM=5nm) into the liquid core bus waveguide with a single mode optical fiber $(4.3 \mu \mathrm{m}$ core diameter). The transmitted light were collected by another single mode optical fiber and sent to a high resolution spectrometer. For an optimized coupling efficiency, an accurate optical alignment between the bus waveguide core and optical fiber has been achieved by employing a micrometer translation stage. Optical measurements were performed by filling the entire device through the open-ended hollow core bus waveguide with methanol and then with DMF by capillary action. The measured total loss of the device with $\mathrm{n}_{\mathrm{c}}=1.32$ is $\sim 22 \mathrm{~dB}$.

The measured normalized transmitted spectrum from the device (methanol-filled core) is shown in Fig.7. From measurements a FSR $=1.62 \mathrm{~nm}$ around $\lambda=640 \mathrm{~nm}$ and an extinction ratio of ER $3.42 \mathrm{~dB}$ were estimated. The measured resonance linewidth (FHWM) is $0.88 \mathrm{~nm}$, leading to a quality factor $\mathrm{Q} \sim 740$. By comparing the simulated and the experimental results we find a good agreement in terms of FSR and ER. The discrepancy between the simulated and experimental quality factor is mainly due to the simplification of the model we used to simulate the device. In fact, by using a two-dimensional model of the device, the modal attenuation of ARROW waveguide due to the vertical confinement is not taken into account. For this reason from simulations result a reduced round-trip ring losses and a larger quality factor $\mathrm{Q}$ with respect to the experimental results.

The optical characterization of the device has been performed also by filling the hollow core with DMF. In Fig. 8 is reported the normalized transmitted spectrum. Form measurements we can note an improvement in terms of extinction ratio, which became ER $\sim 7.64 \mathrm{~dB}$, and also the quality factor $\mathrm{Q} \sim 821$ results slightly increased. Moreover, since a higher refractive index is used, a reduced free spectral range $\mathrm{FSR}=1.55 \mathrm{~nm}$ results from the interference effect in the resonator.

These results are in agreement with the experimental ones and confirm that by reducing the attenuation losses due to the ARROW confinement, an improvement of the ring performances can be obtained. However, in order to get higher Q factor, further improvement of the round-trip losses with respect to the DMF-filled core ring configuration is required. Thanks to the well- known ARROW properties, this can be obtained if only one more bi-layers is added during fabrication procedure to the multilayer stack composing the Fabry-Perot mirror; in this case the attenuation losses of the waveguides are reduced by a factor of 7 [11]. 


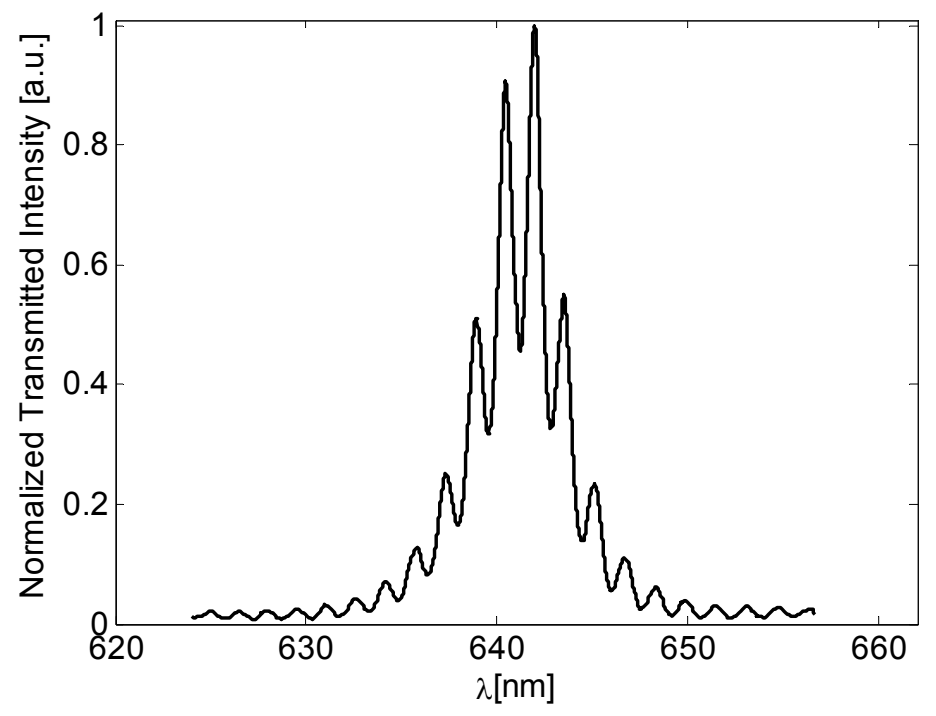

Fig. 7 Normalized transmitted intensity for methanol filled ring resonator $\left(n_{c}=1.32\right)$.

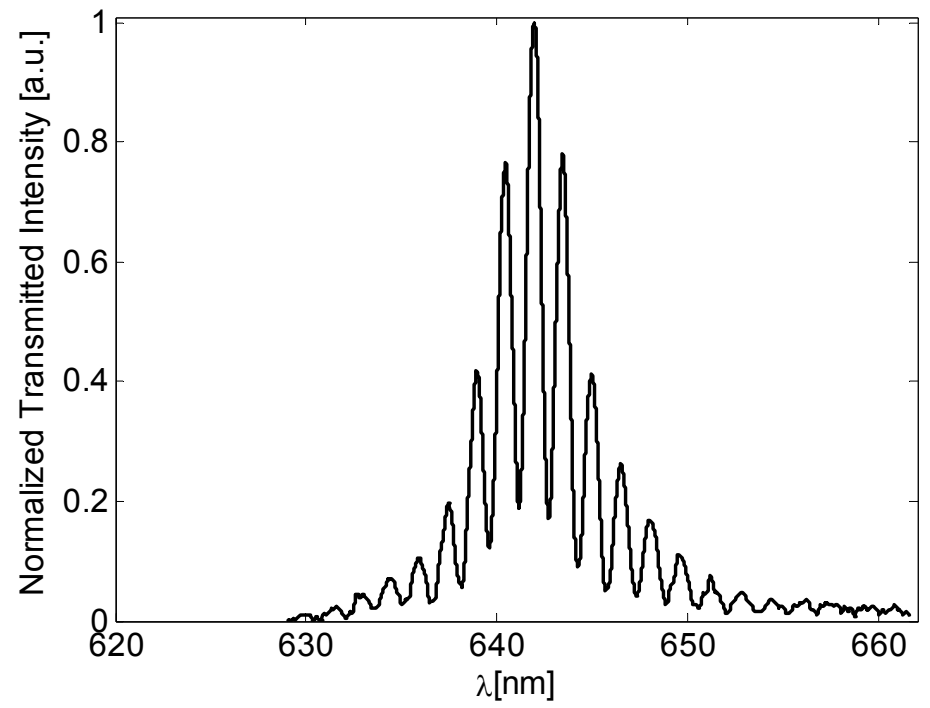

Fig. 8 Normalized transmitted intensity for DMF filled ring resonator $\left(\mathrm{n}_{\mathrm{c}}=1.43\right)$

Finally, we have evaluated the sensing capabilities of the ring. In the case of bulk refractive index sensitivity, the sensitivity is related to the resonance wavelength shift $\Delta \lambda$ due to the change $\Delta \mathrm{n}$ of core refractive index. The wavelength shift can be deduced using the relation: $\Delta \lambda=\lambda \Delta \mathrm{n} / \mathrm{n}$, where $\Delta \mathrm{n} / \mathrm{n}$ was deduced from the free-spectral range measurements. The obtained sensitivity is approximately $260 \mathrm{~nm} / \mathrm{RIU}$. This value is comparable with the theoretical sensitivity of 480 $\mathrm{nm} / \mathrm{RIU}$ obtained by the simulations. This high value can be explained considering that the refractive index sensitivity is governed by the achievable partial sensitivity $\mathrm{Sw}=\partial \mathrm{neff} / \partial \mathrm{nc}$ that takes into account of the optical mode fraction that interacts with the sample. In a liquid core ARROW waveguide the mode field is almost completely confined into the liquid core of the waveguide $(\mathrm{Sw} \approx 1$ ), so the interaction of the sample and field is strongly enhanced as compared to solid core waveguides in which only the evanescent part of the field is sensitive to the surrounding liquid medium. 
In the case of surface sensing, by numerical simulation we have estimated a shift $\Delta \lambda / \lambda$ of about 2e- 8 for a $1 \mathrm{~nm}$ thick layer $(\mathrm{n}=1.45)$. This value is quite low compared to conventional solid core resonator [12], and can be explained taking into account that, in an ARROW waveguide, the mode field intensity at the core-cladding interface is very low and only a very small fraction of the optical mode interacts with the sample.

\section{CONCLUSIONS}

We have demonstrated the feasibility of integrated optofluidic ring resonator. The proposed device has been realized with liquid core ARROW waveguide. With this design the optical part, used to realize the ring, and the microfluidic part, used to deliver the sample, are integrated together. This configuration avoids the need for any additional microfluidics, resulting in a very compact device with a total length of $2.5 \mathrm{~mm}$ and a required liquid volume of about $0.11 \mathrm{nl}$. Numerical simulations have been carried out in order to predict the spectral response of the device for two different refractive index core. Optical characterization of the device has been performed by measuring the spectrum transmitted by the ring filled with methanol and dimethylformamide. We find a good agreement between the numerical simulations and the experimental results. Moreover, from the measured data we derive a very high sensitivity, of about $260 \mathrm{~nm} / \mathrm{RIU}$, in the case of homogeneous sensing. This is due to the strong coupling regime between light and fluid that takes place in a liquid waveguide.

\section{ACKNOWLEDGEMENTS}

The authors would like to thank the IC Process Group of DIMES for technical assistance in the device fabrication.

\section{REFERENCES}

[1] F. Vollmer, D. Braun, A. Libchaber, M. Khoshsima, I. Teraoka, and S. Arnold, "Protein detection by optical shift of a resonant microcavity" Appl. Phys. Lett. 80, 4057-4059 (2002).

[2] C. Chao and L. Guo, "Biochemical sensors based on polymer microrings with sharp asymmetrical resonance", Appl. Phys. Lett. 83, 1527-1529 (2003).

[3] E. Krioukov, D.J. W. Klunder, A. Driessen, J. Greve, and C. Otto, "Integrated optical microcavities for enhanced evanescent-wave spectroscopy", Opt. Lett. 27, 1504-1506 (2002).

[4] A. Nitkowski, L. Chen, and M. Lipson, "Cavity-enhanced on-chip absorption spectroscopy using microring resonators", Opt. Express 16, 11930-11936 (2008).

[5] I. M. White, H. Oveys, and X. Fan, "Liquid-core optical ring-resonator sensors", Opt. Lett. 31, 1319-1321 (2006).

[6] C. F. Carlborg, K. B. Gylfason, A. Kaźmierczak, F. Dortu, M. J. Bañuls Polo, A. Maquieira Catala, G. M. Kresbach, H. Sohlström, T. Moh, L. Vivien, J. Popplewell, G. Ronan, C. A. Barrios, G. Stemme and W. van der Wijngaart, "A packaged optical slot-waveguide ring resonator sensor array for multiplex label-free assays in labson-chips ", Lab Chip 10, 281-290 (2010).

[7] R. Bernini, G. Testa, L. Zeni and P. M. Sarro, "Integrated optofluidic Mach-Zehnder interferometer based on liquid core waveguides", Appl. Phys. Lett. 93, 011106 (2008)

[8] G. Testa, Y. Huang, L. Zeni, P. M. Sarro and R. Bernini, "Liquid Core ARROW Waveguides by Atomic Layer Deposition”, IEEE Photon. Technol. Lett. 22, 616-618 (2010).

[9] H. K. Chiu, F. L. Hsiao, C. H. Chan, C. C. Chen, "Compact and low-loss bent hollow waveguides with distributed Bragg reflector", Opt. Express 16, 15069-15073 (2008).

[10] G. Testa, Y. Huang, P. M. Sarro, L. Zeni, and R. Bernini, "High-visibility optofluidic Mach-Zehnder interferometer", Opt. Lett. 35, 1584-1586 (2010).

[11]H. Schmidt, D. Yin, J. P. Barber, and A. R. Hawkins, "Hollow-core waveguides and 2-D waveguide arrays for integrated optics of gases and liquids", IEEE J. Sel. Top. Quantum Electron, 11, 519-527 (2005).

[12] Vollmer, S. Arnold, "Whispering-gallery-mode biosensing: label-free detection down to single molecules", Nature Methods, 5, 591-596 (2008). 